\title{
An application of matrix inequalities to certain functional inequalities involving fractional powers
}

\section{Keiichi Watanabe*}

\section{"Correspondence:}

wtnbk@math.sc.niigata-u.ac.jp Department of Mathematics,

Faculty of Science, Niigata

University, Niigata, 950-2181, Japan

\section{Abstract}

We will show certain functional inequalities involving fractional powers, making use of the Furuta inequality and Tanahashi's argument.

MSC: Primary 26D07; secondary 26A09; 39B62; 47A63

Keywords: inequalities; fractional powers; matrix inequalities; Furuta inequality

\section{Introduction}

Let $x$ be an arbitrary positive real number. One can easily see the inequality

$$
\left(x^{\frac{3}{2}}-1\right)\left(x^{2}-1\right) \leq \frac{6}{5}\left(x^{\frac{5}{2}}-1\right)(x-1)
$$

for instance, is reduced to a simple polynomial inequality by putting $t=x^{\frac{1}{2}}$. However, at least to the author, it seems not easy to give an elementary proof of the inequality

$$
x^{\frac{2-\sqrt{2}+\sqrt{3}}{4}}\left(x^{\sqrt{2}}-1\right)\left(x^{\frac{\sqrt{2}+\sqrt{3}}{2}}-1\right) \leq \frac{1}{\sqrt{2}}\left(x^{\sqrt{2}+\sqrt{3}}-1\right)(x-1),
$$

which has a very similar form to the preceding one although their corresponding numerical parts are different.

The purpose of this article is to show the following theorem.

Theorem 1.1 Let $0 \leq p, 1 \leq q$ and $0 \leq r$ with $p+r \leq(1+r) q$. If $0<x$, then

$$
x^{\frac{1+r-\frac{p+r}{q}}{2}}\left(x^{p}-1\right)\left(x^{\frac{p+r}{q}}-1\right) \leq \frac{p}{q}\left(x^{p+r}-1\right)(x-1) .
$$

An elementary approach to proving the inequality (1) might be to consider the power series expansion.

$$
\begin{aligned}
& \text { Put } t=x-1, c=\frac{1+r-\frac{p+r}{q}}{2} \text { and } \\
& \qquad f(t)=\frac{p}{q}\left((1+t)^{p+r}-1\right) t-(1+t)^{c}\left((1+t)^{p}-1\right)\left((1+t)^{\frac{p+r}{q}}-1\right) .
\end{aligned}
$$

(0) 2012 Watanabe; licensee Springer. This is an Open Access article distributed under the terms of the Creative Commons Attribution License (http://creativecommons.org/licenses/by/2.0), which permits unrestricted use, distribution, and reproduction in any medium, provided the original work is properly cited. 
Then we can expand $f(t)$ around $t=0$ as

$$
\begin{aligned}
f(t)= & \frac{p}{q}\left\{p+r+\left(\begin{array}{c}
p+r \\
2
\end{array}\right) t+\left(\begin{array}{c}
p+r \\
3
\end{array}\right) t^{2}+\left(\begin{array}{c}
p+r \\
4
\end{array}\right) t^{3}+\left(\begin{array}{c}
p+r \\
5
\end{array}\right) t^{4}+\cdots\right\} \cdot t^{2} \\
& -\left\{1+c t+\left(\begin{array}{c}
c \\
2
\end{array}\right) t^{2}+\left(\begin{array}{c}
c \\
3
\end{array}\right) t^{3}+\left(\begin{array}{c}
c \\
4
\end{array}\right) t^{4}+\cdots\right\} \\
& \cdot\left\{p+\left(\begin{array}{c}
p \\
2
\end{array}\right) t+\left(\begin{array}{c}
p \\
3
\end{array}\right) t^{2}+\left(\begin{array}{c}
p \\
4
\end{array}\right) t^{3}+\left(\begin{array}{c}
p \\
5
\end{array}\right) t^{4}+\cdots\right\} \\
& \cdot\left\{\frac{p+r}{q}+\left(\begin{array}{c}
\frac{p+r}{q} \\
2
\end{array}\right) t+\left(\begin{array}{c}
\frac{p+r}{q} \\
3
\end{array}\right) t^{2}+\left(\begin{array}{c}
\frac{p+r}{q} \\
4
\end{array}\right) t^{3}+\left(\begin{array}{c}
\frac{p+r}{q} \\
5
\end{array}\right) t^{4}+\cdots\right\} \cdot t^{2} \\
= & a_{4} t^{4}+a_{5} t^{5}+a_{6} t^{6}+\cdots
\end{aligned}
$$

Thus, the constant term and the coefficients of $t, t^{2}$ and $t^{3}$ are 0 . Further, one can obtain

$$
\begin{aligned}
& a_{4}=\frac{p(p+r)}{24 q}\left(r^{2}+2 p r+1-\left(\frac{p+r}{q}\right)^{2}\right), \\
& a_{5}=\frac{p(p+r)(p+r-3)}{48 q}\left(r^{2}+2 p r+1-\left(\frac{p+r}{q}\right)^{2}\right)
\end{aligned}
$$

and

$$
\begin{aligned}
a_{6}= & -\frac{p(p+r)}{5760 q}\left\{3\left(\frac{p+r}{q}\right)^{4}+10\left(\frac{p+r}{q}\right)^{2}\left\{3(p+r)(p+r-8)+p^{2}+41\right\}\right. \\
& -33(p+r)^{4}+240(p+r)^{3}+30(p+r)^{2}\left(p^{2}-15\right)-240(p+r)\left(p^{2}-1\right) \\
& \left.+\left(3 p^{2}+413\right)\left(p^{2}-1\right)\right\} .
\end{aligned}
$$

Thus, if the assumption for the parameters $p, q$ and $r$ in Theorem 1.1 is satisfied, then we have $0<a_{4}$. However, the signature of $a_{5}$ and $a_{6}$ depends on parameters, and one cannot see any signs of a simple rule among the coefficients of higher order terms. Although $f(t)$ is non-negative on a sufficiently small neighborhood of $t=0$, it seems difficult to show that $f(t)$ is non-negative entirely on $-1<t<\infty$ by such an argument as above.

Let us recall some fundamental concepts on related matrix inequalities. A capital letter means a matrix whose entries are complex numbers. A square matrix $T$ is said to be positive semidefinite (denoted by $0 \leq T)$ if $0 \leq(T x, x)$ for all vectors $x$. We write $0<T$ if $T$ is positive semidefinite and invertible. For two selfadjoint matrices $T_{1}$ and $T_{2}$ of the same size, a matrix inequality $T_{1} \leq T_{2}$ is defined by $0 \leq T_{2}-T_{1}$.

The celebrated Löwner-Heinz theorem includes:

Theorem 1.2 [1,2] Let $0 \leq p \leq 1$. If $0 \leq B \leq A$, then $B^{p} \leq A^{p}$.

For $1<p, 0 \leq B \leq A$ does not always ensure $B^{p} \leq A^{p}$. Furuta obtained an epoch-making extension of the Löwner-Heinz inequality by using the Löwner-Heinz inequality itself.

Theorem 1.3 [3] Let $0 \leq p, 1 \leq q$ and $0 \leq r$ with $p+r \leq(1+r) q$. If $0 \leq B \leq A$, then

$$
\left(A^{\frac{r}{2}} B^{p} A^{\frac{r}{2}}\right)^{\frac{1}{q}} \leq A^{\frac{p+r}{q}} .
$$


The following result by Tanahashi is a full description of the best possibility of the range

$$
p+r \leq(1+r) q \text { and } 1 \leq q
$$

as far as all parameters are positive.

Theorem 1.4 [4] Let $p, q, r$ be positive real numbers. If $(1+r) q<p+r$ or $0<q<1$, then there exist $2 \times 2$ matrices $A, B$ with $0<B \leq A$ that do not satisfy the inequality

$$
\left(A^{\frac{r}{2}} B^{p} A^{\frac{r}{2}}\right)^{\frac{1}{q}} \leq A^{\frac{p+r}{q}} .
$$

One notices the coincidence between the assumption on parameters in Theorem 1.1 and Theorem 1.3. As a matter of fact, the inequality (1) is a particular conclusion of the Furuta inequality. We should point out that Tanahashi's argument in [4] is almost sufficient to deduce the former from the latter. In the next section, we will prove Theorem $1.1 \mathrm{using}$ Theorem 1.3 and Tanahashi's argument.

\section{Proof of Theorem 1.1}

As we mentioned above, our proof of Theorem 1.1 has a major part which is parallel to [4]. Our matrix $A$ is a little different from that in [4], we use a variable $y$ instead of $\varepsilon$ and $\delta$. It simplifies the argument to an extent, though the improvement is not essential.

Throughout this paper, we assume that $1<a<b$ and $0<y$. We will consider matrices

$$
A=\left(\begin{array}{cc}
a & \sqrt{(a-1) y} \\
\sqrt{(a-1) y} & b+y
\end{array}\right)
$$

and

$$
B=\left(\begin{array}{ll}
1 & 0 \\
0 & b
\end{array}\right)
$$

Then we have $0<B \leq A$. The eigenvalues of $A$ are $\frac{a+b+y \pm \sqrt{d}}{2}$, where $d=a^{2}+b^{2}+y^{2}-$ $2 a b+2(a+b-2) y$.

Lemma $2.10<d<(a+b+y)^{2}$ and $a-b-y-\sqrt{d} \neq 0$.

Proof Obviously,

$$
\begin{aligned}
& d=(a-b)^{2}+y(y+2(a+b-2))>0, \\
& d=(a+b+y)^{2}-4(a b+y)<(a+b+y)^{2} .
\end{aligned}
$$

If $a-b-y-\sqrt{d}=0$, then we would have $a=1$ or $y=0$, which is contrary to the assumption.

Let

$$
c=\frac{-2 \sqrt{(a-1) y}}{a-b-y-\sqrt{d}}
$$


and

$$
U=\frac{1}{\sqrt{c^{2}+1}}\left(\begin{array}{cc}
c & 1 \\
1 & -c
\end{array}\right)
$$

Then $U$ is unitary and

$$
U^{*} A U=\frac{1}{2}\left(\begin{array}{ll}
d_{1} & 0 \\
0 & d_{2}
\end{array}\right)
$$

where

$$
d_{1}=a+b+y+\sqrt{d}, \quad d_{2}=a+b+y-\sqrt{d} .
$$

By the assumption and Theorem 1.3, $A$ and $B$ satisfy the inequality (2). Then

$$
\left(U^{*} A^{\frac{r}{2}} U U^{*} B^{p} U U^{*} A^{\frac{r}{2}} U\right)^{\frac{1}{q}} \leq U^{*} A^{\frac{p+r}{q}} U,
$$

hence we have

$$
\left\{\left(\begin{array}{cc}
d_{1}^{\frac{r}{2}} & 0 \\
0 & d_{2}^{\frac{r}{2}}
\end{array}\right) U^{*}\left(\begin{array}{cc}
1 & 0 \\
0 & b^{p}
\end{array}\right) U\left(\begin{array}{cc}
d_{1}^{\frac{r}{2}} & 0 \\
0 & d_{2}^{\frac{r}{2}}
\end{array}\right)\right\}^{\frac{1}{q}} \leq 2^{-\frac{p}{q}}\left(\begin{array}{cc}
d_{1}^{\frac{p+r}{q}} & 0 \\
0 & d_{2}^{\frac{p+r}{q}}
\end{array}\right)
$$

Denote

$$
\left(\begin{array}{cc}
d_{1}^{\frac{r}{2}} & 0 \\
0 & d_{2}^{\frac{r}{2}}
\end{array}\right) U^{*}\left(\begin{array}{cc}
1 & 0 \\
0 & b^{p}
\end{array}\right) U\left(\begin{array}{cc}
d_{1}^{\frac{r}{2}} & 0 \\
0 & d_{2}^{\frac{r}{2}}
\end{array}\right)=\frac{1}{c^{2}+1}\left(\begin{array}{cc}
A_{1} & A_{3} \\
A_{3} & A_{2}
\end{array}\right)
$$

where

$$
\begin{aligned}
& A_{1}=d_{1}^{r}\left(c^{2}+b^{p}\right), \\
& A_{2}=d_{2}^{r}\left(1+c^{2} b^{p}\right), \\
& A_{3}=d_{1}^{\frac{r}{2}} d_{2}^{\frac{r}{2}} c\left(1-b^{p}\right)=\left((a+b+y)^{2}-d\right)^{\frac{r}{2}} c\left(1-b^{p}\right)=(4 a b+4 y)^{\frac{r}{2}} c\left(1-b^{p}\right) .
\end{aligned}
$$

Lemma 2.2 Let $p, q, r$ be positive real numbers. Then $A_{2}<A_{1}$ and $A_{3}<0$.

Proof Since $d_{2}<d_{1}$ and $0<r$, we have $d_{2}^{r}<d_{1}^{r}$. Moreover,

$$
\left(c^{2}+b^{p}\right)-\left(1+c^{2} b^{p}\right)=\left(c^{2}-1\right)\left(1-b^{p}\right), \quad 1-b^{p}<0
$$

and

$$
c^{2}-1=-\frac{2(a-b)^{2}+2 y^{2}+4(b-a) y+2(b-a+y) \sqrt{d}}{(a-b-y-\sqrt{d})^{2}}<0,
$$

hence we have $1+c^{2} b^{p}<c^{2}+b^{p}$. Thus $A_{2}<A_{1}$. 
It is obvious that $1-b^{p}<0$ and $0<c$, and hence $A_{3}<0$.

Let

$$
V=\frac{1}{\sqrt{A_{1}-A_{2}+2 \varepsilon_{1}}}\left(\begin{array}{cc}
\sqrt{A_{1}-A_{2}+\varepsilon_{1}} & -\sqrt{\varepsilon_{1}} \\
-\sqrt{\varepsilon_{1}} & -\sqrt{A_{1}-A_{2}+\varepsilon_{1}}
\end{array}\right),
$$

where

$$
2 \varepsilon_{1}=-A_{1}+A_{2}+\sqrt{\left(A_{1}-A_{2}\right)^{2}+4 A_{3}^{2}} .
$$

Then it is easy to see that $A_{3}=-\sqrt{\left(A_{1}-A_{2}+\varepsilon_{1}\right) \varepsilon_{1}}, V$ is unitary and

$$
V^{*}\left(\begin{array}{ll}
A_{1} & A_{3} \\
A_{3} & A_{2}
\end{array}\right) V=\left(\begin{array}{cc}
A_{1}+\varepsilon_{1} & 0 \\
0 & A_{2}-\varepsilon_{1}
\end{array}\right)
$$

The following lemma is one of the most important points in Tanahashi's argument. Although the substance is presented in the whole proof of [4, Theorem], we should restate and prove it in our context for the readers' convenience.

\section{Lemma 2.3}

$$
\begin{aligned}
& \varepsilon_{1}\left\{\gamma d_{1}^{\frac{p+r}{q}}-\left(A_{2}-\varepsilon_{1}\right)^{\frac{1}{q}}\right\}\left\{\left(A_{1}+\varepsilon_{1}\right)^{\frac{1}{q}}-\gamma d_{2}^{\frac{p+r}{q}}\right\} \\
& \quad \leq\left(A_{1}-A_{2}+\varepsilon_{1}\right)\left\{\gamma d_{1}^{\frac{p+r}{q}}-\left(A_{1}+\varepsilon_{1}\right)^{\frac{1}{q}}\right\}\left\{\gamma d_{2}^{\frac{p+r}{q}}-\left(A_{2}-\varepsilon_{1}\right)^{\frac{1}{q}}\right\},
\end{aligned}
$$

where $\gamma=\left(\frac{c^{2}+1}{2^{p}}\right)^{\frac{1}{q}}$.

Proof The formula (3) implies

$$
\left(c^{2}+1\right)^{-\frac{1}{q}} V\left(\begin{array}{cc}
\left(A_{1}+\varepsilon_{1}\right)^{\frac{1}{q}} & 0 \\
0 & \left(A_{2}-\varepsilon_{1}\right)^{\frac{1}{q}}
\end{array}\right) V^{*} \leq 2^{-\frac{p}{q}}\left(\begin{array}{cc}
d_{1}^{\frac{p+r}{q}} & 0 \\
0 & d_{2}^{\frac{p+r}{q}}
\end{array}\right) .
$$

Write the left-hand matrix as

$$
\left(c^{2}+1\right)^{-\frac{1}{q}}\left(A_{1}-A_{2}+2 \varepsilon_{1}\right)^{-1}\left(\begin{array}{cc}
B_{1} & B_{3} \\
B_{3} & B_{2}
\end{array}\right)
$$

where

$$
\begin{aligned}
& B_{1}=\left(A_{1}-A_{2}+\varepsilon_{1}\right)\left(A_{1}+\varepsilon_{1}\right)^{\frac{1}{q}}+\varepsilon_{1}\left(A_{2}-\varepsilon_{1}\right)^{\frac{1}{q}}, \\
& B_{2}=\varepsilon_{1}\left(A_{1}+\varepsilon_{1}\right)^{\frac{1}{q}}+\left(A_{1}-A_{2}+\varepsilon_{1}\right)\left(A_{2}-\varepsilon_{1}\right)^{\frac{1}{q}}, \\
& B_{3}=-\sqrt{A_{1}-A_{2}+\varepsilon_{1}} \sqrt{\varepsilon_{1}}\left\{\left(A_{1}+\varepsilon_{1}\right)^{\frac{1}{q}}-\left(A_{2}-\varepsilon_{1}\right)^{\frac{1}{q}}\right\} .
\end{aligned}
$$

Then, by the formula (5), we have

$$
0 \leq\left(\begin{array}{cc}
\gamma\left(A_{1}-A_{2}+2 \varepsilon_{1}\right) d_{1}^{\frac{p+r}{q}}-B_{1} & -B_{3} \\
-B_{3} & \gamma\left(A_{1}-A_{2}+2 \varepsilon_{1}\right) d_{2}^{\frac{p+r}{q}}-B_{2}
\end{array}\right)
$$


So, its determinant is also non-negative. We expand it to obtain

$$
\begin{aligned}
0 \leq & \gamma^{2}\left(A_{1}-A_{2}+2 \varepsilon_{1}\right)^{2} d_{1}^{\frac{p+r}{q}} d_{2}^{\frac{p+r}{q}}-\gamma\left(A_{1}-A_{2}+2 \varepsilon_{1}\right) d_{1}^{\frac{p+r}{q}} B_{2} \\
& -\gamma\left(A_{1}-A_{2}+2 \varepsilon_{1}\right) d_{2}^{\frac{p+r}{q}} B_{1}+B_{1} B_{2}-B_{3}^{2} .
\end{aligned}
$$

Now,

$$
\begin{aligned}
B_{1} B_{2} & -B_{3}^{2} \\
= & \left\{\left(A_{1}-A_{2}+\varepsilon_{1}\right)\left(A_{1}+\varepsilon_{1}\right)^{\frac{1}{q}}+\varepsilon_{1}\left(A_{2}-\varepsilon_{1}\right)^{\frac{1}{q}}\right\}\left\{\varepsilon_{1}\left(A_{1}+\varepsilon_{1}\right)^{\frac{1}{q}}+\left(A_{1}-A_{2}+\varepsilon_{1}\right)\left(A_{2}-\varepsilon_{1}\right)^{\frac{1}{q}}\right\} \\
& -\left(A_{1}-A_{2}+\varepsilon_{1}\right) \varepsilon_{1}\left\{\left(A_{1}+\varepsilon_{1}\right)^{\frac{1}{q}}-\left(A_{2}-\varepsilon_{1}\right)^{\frac{1}{q}}\right\}^{2} \\
= & \left(A_{1}-A_{2}+2 \varepsilon_{1}\right)^{2}\left(A_{1}+\varepsilon_{1}\right)^{\frac{1}{q}}\left(A_{2}-\varepsilon_{1}\right)^{\frac{1}{q}} .
\end{aligned}
$$

Hence, the formula (6) implies

$$
\begin{aligned}
0 \leq & \left(A_{1}-A_{2}+2 \varepsilon_{1}\right)\left\{\gamma^{2}\left(A_{1}-A_{2}+2 \varepsilon_{1}\right) d_{1}^{\frac{p+r}{q}} d_{2}^{\frac{p+r}{q}}-\gamma d_{1}^{\frac{p+r}{q}} B_{2}-\gamma d_{2}^{\frac{p+r}{q}} B_{1}\right\} \\
& +\left(A_{1}-A_{2}+2 \varepsilon_{1}\right)^{2}\left(A_{1}+\varepsilon_{1}\right)^{\frac{1}{q}}\left(A_{2}-\varepsilon_{1}\right)^{\frac{1}{q}} .
\end{aligned}
$$

Cancel the common positive factor $A_{1}-A_{2}+2 \varepsilon_{1}$ and substitute the definitions for $B_{1}$ and $B_{2}$. Then a simple calculation shows that

$$
\begin{aligned}
-\varepsilon_{1} & \left\{\gamma^{2} d_{1}^{\frac{p+r}{q}} d_{2}^{\frac{p+r}{q}}-\gamma d_{1}^{\frac{p+r}{q}}\left(A_{1}+\varepsilon_{1}\right)^{\frac{1}{q}}-\gamma d_{2}^{\frac{p+r}{q}}\left(A_{2}-\varepsilon_{1}\right)^{\frac{1}{q}}+\left(A_{1}+\varepsilon_{1}\right)^{\frac{1}{q}}\left(A_{2}-\varepsilon_{1}\right)^{\frac{1}{q}}\right\} \\
\leq & \left(A_{1}-A_{2}+\varepsilon_{1}\right) \\
& \cdot\left\{\gamma^{2} d_{1}^{\frac{p+r}{q}} d_{2}^{\frac{p+r}{q}}-\gamma d_{1}^{\frac{p+r}{q}}\left(A_{2}-\varepsilon_{1}\right)^{\frac{1}{q}}-\gamma d_{2}^{\frac{p+r}{q}}\left(A_{1}+\varepsilon_{1}\right)^{\frac{1}{q}}+\left(A_{1}+\varepsilon_{1}\right)^{\frac{1}{q}}\left(A_{2}-\varepsilon_{1}\right)^{\frac{1}{q}}\right\} .
\end{aligned}
$$

By factorizing, we have

$$
\begin{aligned}
& -\varepsilon_{1}\left\{\gamma d_{1}^{\frac{p+r}{q}}-\left(A_{2}-\varepsilon_{1}\right)^{\frac{1}{q}}\right\}\left\{\gamma d_{2}^{\frac{p+r}{q}}-\left(A_{1}+\varepsilon_{1}\right)^{\frac{1}{q}}\right\} \\
& \quad \leq\left(A_{1}-A_{2}+\varepsilon_{1}\right)\left\{\gamma d_{1}^{\frac{p+r}{q}}-\left(A_{1}+\varepsilon_{1}\right)^{\frac{1}{q}}\right\}\left\{\gamma d_{2}^{\frac{p+r}{q}}-\left(A_{2}-\varepsilon_{1}\right)^{\frac{1}{q}}\right\} .
\end{aligned}
$$

This completes the proof of Lemma 2.3 .

Now, we estimate each term of the inequality (4) with respect to $y \rightarrow+0$. A key point in making use of the inequality (4) is that both estimations of the factor $\varepsilon_{1}$ on the left-hand side and the factor $\gamma d_{1}^{\frac{p+r}{q}}-\left(A_{1}+\varepsilon_{1}\right)^{\frac{1}{q}}$ on the right-hand side contain a common subfactor $y$. After the cancellation of this $y$, we will derive the desired functional inequality by letting $y \rightarrow+0, a \rightarrow 1+0$ and applying l'Hopital's rule. Terms in other factors can be roughly estimated.

In the following, $o$ means $o(y)$, that is

$$
\frac{o}{y} \rightarrow 0 \quad(y \rightarrow+0)
$$


and $o(1)$ denotes a term such that $o(1) \rightarrow 0(y \rightarrow+0)$.

One can establish the following formulae:

$$
\begin{aligned}
& \sqrt{d}=(b-a)\left\{1+\frac{a+b-2}{(b-a)^{2}} y+o(y)\right\} \\
& d_{1}^{\frac{p+r}{q}}=(2 b)^{\frac{p+r}{q}}\left\{1+\frac{p+r}{q} \cdot \frac{b-1}{b(b-a)} y+o(y)\right\} \text {, } \\
& d_{2}^{\frac{p+r}{q}}=(2 a)^{\frac{p+r}{q}}\left\{1+\frac{p+r}{q} \cdot \frac{-a+1}{a(b-a)} y+o(y)\right\} \text {, } \\
& c=\frac{-2 \sqrt{(a-1) y}}{a-b-y-\left(b-a+\frac{a+b-2}{b-a} y+o(y)\right)}=\sqrt{y} \cdot \frac{\sqrt{a-1}}{b-a}\left\{1-\frac{b-1}{(b-a)^{2}} y+o(y)\right\}, \\
& c^{2}+1=1+\frac{a-1}{(b-a)^{2}} y+o(y), \\
& \left(c^{2}+1\right)^{\frac{1}{q}} d_{1}^{\frac{p+r}{q}} \\
& =\left\{1+\frac{a-1}{q(b-a)^{2}} y+o(y)\right\}(2 b)^{\frac{p+r}{q}}\left\{1+\frac{p+r}{q} \cdot \frac{b-1}{b(b-a)} y+o(y)\right\} \\
& =(2 b)^{\frac{p+r}{q}}\left\{1+\frac{1}{q b(b-a)^{2}}((a-1) b+(p+r)(b-1)(b-a)) y+o(y)\right\} \text {, } \\
& \left(c^{2}+1\right)^{\frac{1}{q}} d_{2}^{\frac{p+r}{q}}=(2 a)^{\frac{p+r}{q}}(1+o(1)) \text {, } \\
& A_{1}=(2 b)^{r}\left\{1+\frac{r(b-1)}{b(b-a)} y+o(y)\right\}\left\{b^{p}+\frac{a-1}{(b-a)^{2}} y+o(y)\right\} \\
& =2^{r} b^{p+r}\left\{1+\frac{1}{b(b-a)^{2}}\left(r(b-1)(b-a)+b^{1-p}(a-1)\right) y+o(y)\right\}, \\
& A_{2}=(2 a)^{r}(1+o(1)) \text {, } \\
& A_{3}^{2}=(4 a b+4 y)^{r} y \frac{a-1}{(b-a)^{2}}(1+o(1))\left(1-b^{p}\right)^{2}=y 4^{r} a^{r} b^{r} \frac{a-1}{(b-a)^{2}}\left(1-b^{p}\right)^{2}(1+o(1)), \\
& \varepsilon_{1}=\frac{1}{2}\left(A_{1}-A_{2}\right)\left(-1+\sqrt{1+\frac{4 A_{3}^{2}}{\left(A_{1}-A_{2}\right)^{2}}}\right)=\frac{A_{3}^{2}}{A_{1}-A_{2}}+o \\
& =\frac{y 4^{r} a^{r} b^{r}(a-1)(b-a)^{-2}\left(1-b^{p}\right)^{2}(1+o(1))}{2^{r} b^{p+r}(1+o(1))-(2 a)^{r}(1+o(1))}+o \\
& =\frac{y 2^{r} a^{r} b^{r}(a-1)\left(1-b^{p}\right)^{2}}{(b-a)^{2}\left(b^{p+r}-a^{r}\right)}(1+o(1)) \text {, } \\
& \left(A_{1}+\varepsilon_{1}\right)^{\frac{1}{q}} \\
& =\left(2^{r} b^{p+r}\left\{1+\frac{1}{b(b-a)^{2}}\left(r(b-1)(b-a)+b^{1-p}(a-1)\right) y+o(y)\right\}\right. \\
& \left.+\frac{y 2^{r} a^{r} b^{r}(a-1)\left(1-b^{p}\right)^{2}}{(b-a)^{2}\left(b^{p+r}-a^{r}\right)}(1+o(1))\right)^{\frac{1}{q}} \\
& =2^{\frac{r}{q}} b^{\frac{p+r}{q}}\left\{1+\frac{1}{q b(b-a)^{2}}\left(r(b-1)(b-a)+b^{1-p}(a-1)+\frac{a^{r} b^{1-p}(a-1)\left(1-b^{p}\right)^{2}}{b^{p+r}-a^{r}}\right) y\right. \\
& +o(y)\} \text {, }
\end{aligned}
$$




$$
\begin{aligned}
& \left(A_{2}-\varepsilon_{1}\right)^{\frac{1}{q}}=2^{\frac{r}{q}} a^{\frac{r}{q}}(1+o(1)), \\
& A_{1}-A_{2}+\varepsilon_{1}=2^{r}\left(b^{p+r}-a^{r}\right)(1+o(1)), \\
& \gamma d_{1}^{\frac{p+r}{q}}-\left(A_{2}-\varepsilon_{1}\right)^{\frac{1}{q}}=2^{\frac{r}{q}}\left(b^{\frac{p+r}{q}}-a^{\frac{r}{q}}\right)(1+o(1)), \\
& \gamma d_{2}^{\frac{p+r}{q}}-\left(A_{1}+\varepsilon_{1}\right)^{\frac{1}{q}}=2^{\frac{r}{q}}\left(a^{\frac{p+r}{q}}-b^{\frac{p+r}{q}}\right)(1+o(1)), \\
& \gamma d_{2}^{\frac{p+r}{q}}-\left(A_{2}-\varepsilon_{1}\right)^{\frac{1}{q}}=2^{\frac{r}{q}}\left(a^{\frac{p+r}{q}}-a^{\frac{r}{q}}\right)(1+o(1)) .
\end{aligned}
$$

Now, we have the estimation of the most delicate factor in the formula (4), whose constant term is canceled by subtraction.

$$
\begin{aligned}
\gamma d_{1}^{\frac{p+r}{q}} & -\left(A_{1}+\varepsilon_{1}\right)^{\frac{1}{q}} \\
= & 2^{-\frac{p}{q}} \cdot(2 b)^{\frac{p+r}{q}}\left\{1+\frac{1}{q b(b-a)^{2}}((a-1) b+(p+r)(b-1)(b-a)) y+o(y)\right\} \\
& -2^{\frac{r}{q}} b^{\frac{p+r}{q}}\left\{1+\frac{1}{q b(b-a)^{2}}\left(r(b-1)(b-a)+b^{1-p}(a-1)+\frac{a^{r} b^{1-p}(a-1)\left(1-b^{p}\right)^{2}}{b^{p+r}-a^{r}}\right) y\right. \\
& +o(y)\} \\
= & 2^{\frac{r}{q}} \frac{b^{\frac{p+r}{q}-1}}{q(b-a)^{2}}\left\{(a-1) b+p(b-1)(b-a)-b^{1-p}(a-1)-\frac{a^{r} b^{1-p}(a-1)\left(1-b^{p}\right)^{2}}{b^{p+r}-a^{r}}\right\} y \\
& \cdot(1+o(1))
\end{aligned}
$$

Substitute these estimations for the inequality (4), cancel the positive factor $y$, and let $y \rightarrow+0$, then we have

$$
\begin{aligned}
& \frac{2^{r} a^{r} b^{r}(a-1)\left(1-b^{p}\right)^{2}}{(b-a)^{2}\left(b^{p+r}-a^{r}\right)} \cdot 2^{\frac{r}{q}}\left(b^{\frac{p+r}{q}}-a^{\frac{r}{q}}\right) \cdot 2^{\frac{r}{q}}\left(b^{\frac{p+r}{q}}-a^{\frac{p+r}{q}}\right) \\
& \leq 2^{r}\left(b^{p+r}-a^{r}\right) \\
& \quad \cdot 2^{\frac{r}{q}} \frac{b^{\frac{p+r}{q}-1}}{q(b-a)^{2}}\left\{(a-1) b+p(b-1)(b-a)-b^{1-p}(a-1)-\frac{a^{r} b^{1-p}(a-1)\left(1-b^{p}\right)^{2}}{b^{p+r}-a^{r}}\right\} \\
& \quad \cdot 2^{\frac{r}{q}}\left(a^{\frac{p+r}{q}}-a^{\frac{r}{q}}\right),
\end{aligned}
$$

and hence

$$
\begin{aligned}
a^{r} b^{r}( & \left.1-b^{p}\right)^{2} \cdot\left(b^{\frac{p+r}{q}}-a^{\frac{r}{q}}\right) \cdot\left(b^{\frac{p+r}{q}}-a^{\frac{p+r}{q}}\right) \\
\leq & \left(b^{p+r}-a^{r}\right)^{2} \\
& \quad \frac{b^{\frac{p+r}{q}-1}}{q}\left\{(a-1) b+p(b-1)(b-a)-b^{1-p}(a-1)-\frac{a^{r} b^{1-p}(a-1)\left(1-b^{p}\right)^{2}}{b^{p+r}-a^{r}}\right\} \\
& \quad \frac{a^{\frac{p+r}{q}}-a^{\frac{r}{q}}}{a-1} .
\end{aligned}
$$


Letting $a \rightarrow 1+0$ and applying l'Hopital's rule, we have

$$
b^{r}\left(1-b^{p}\right)^{2}\left(b^{\frac{p+r}{q}}-1\right)^{2} \leq\left(b^{p+r}-1\right)^{2} b^{\frac{p+r}{q}-1} \frac{p^{2}}{q^{2}}(b-1)^{2} .
$$

This implies that, for arbitrary $1<b$,

$$
b^{\frac{1+r-\frac{p+r}{q}}{2}}\left(b^{p}-1\right)\left(b^{\frac{p+r}{q}}-1\right) \leq \frac{p}{q}\left(b^{p+r}-1\right)(b-1) .
$$

For arbitrary $0<x<1$, substitute $\frac{1}{x}$ for $b$ in (7) and multiply by $x, x^{p}, x^{p+r}, x^{\frac{p+r}{q}}$ both sides. It is easy to see that $x$ itself satisfies (7). This completes the proof of Theorem 1.1.

\section{Competing interests}

The author declares that he has no competing interests.

\section{Acknowledgements}

The author was supported in part by Grants-in-Aid for Scientific Research, Japan Society for the Promotion of Science.

Received: 9 May 2012 Accepted: 20 September 2012 Published: 3 October 2012

\section{References}

1. Löwner, K: Über monotone Matrixfunktionen. Math. Z. 38, 177-216 (1934)

2. Heinz, E: Beiträge zur Störungstheorie der Spektralzerlegung. Math. Ann. 123, 415-438 (1951)

3. Furuta, $\mathrm{T}: A \geq B \geq 0$ assures $\left(B^{r} A^{p} B^{r}\right)^{1 / q} \geq B^{(p+2 r) / q}$ for $r \geq 0, p \geq 0, q \geq 1$ with $(1+2 r) q \geq p+2 r$. Proc. Am. Math. Soc 101(1), 85-88 (1987)

4. Tanahashi, K: Best possibility of the Furuta inequality. Proc. Am. Math. Soc. 124, 141-146 (1996)

doi:10.1186/1029-242X-2012-221

Cite this article as: Watanabe: An application of matrix inequalities to certain functional inequalities involving

fractional powers. Journal of Inequalities and Applications 2012 2012:221.

\section{Submit your manuscript to a SpringerOpen ${ }^{\ominus}$ journal and benefit from:}

- Convenient online submission

- Rigorous peer review

- Immediate publication on acceptance

- Open access: articles freely available online

- High visibility within the field

- Retaining the copyright to your article 